\title{
Transcatheter closure of atrial septal defect with Chinese and Thai nitinol wire mesh occluders in adult patients
}

\author{
Filip Tyc' ${ }^{1}$, Alexander Suchodolski1, Mateusz Knop², Dominika Rojczyk², Michał Gałeczka², \\ Sebastian Smerdziński², Małgorzata Szkutnik², Jacek Białkowski², Roland Fiszer ${ }^{2}$ \\ 1 Student Research Group, Department of Congenital Heart Diseases and Pediatric Cardiology, School of Medicine with Division \\ of Dentistry in Zabrze, Medical University of Silesia, Silesian Centre for Heart Diseases, Zabrze, Poland \\ 2 Department of Congenital Heart Diseases and Pediatric Cardiology, School of Medicine with Division of Dentistry \\ in Zabrze, Medical University of Silesia, Silesian Centre for Heart Diseases, Zabrze, Poland
}

Correspondence to: Jacek Białkowski, MD, PhD, Department of Congenital Heart Diseases and Pediatric Cardiology, Silesian Centre for Heart Diseases, ul. Marii Curie-Skłodowskiej 9 , 41-800 Zabrze, Poland, phone: +48322713401, email:jabi_med@poczta.onet.pl Received: October 15, 2019 Revision accepted: January 3, 2020. Published online: January 4, 2020. Kardiol Pol. 2020; 78 (1): 68-70 doi:10.33963/KP.15121 Copyright by the Author(s), 2020
Introduction Transcatheter closure of atrial septal defect (ASD) type II can be safely performed in children and adults. ${ }^{1,2}$ Since the implementation of the Amplatzer septal occluder (ASO) in 1997, many similar nitinol wire mesh Amplatzer-like occluders have been developed. Nitinol is an alloy of nickel and titanium. Studies revealed a rise in nickel serum concentration, especially in the first 3 months after the ASO implantation. ${ }^{3,4}$ To prevent this phenomenon associated with the use of several Amplatzer-like occluders (also the ASO from 2014), different methods to pretreat nitinol wires were applied.

The literature on the use of Chinese and Thai occluders for percutaneous ASD closure is scarce. ${ }^{5}$ All these Amplatzer-like occluders are very similar in shape, dimensions, structure of the delivery system, and the technique of implantation.

We described our experience with ASD closure in adults patients with the ASO devices elsewhere. ${ }^{6}$ However, to our best knowledge, this is the first study on ASD closure with the use of different nitinol wire devices (Chinese and Thai) with and without a protective nitinol layer.

Methods Between 1997 and 2018, over 1800 patients (children and adults) underwent transcatheter closure of interatrial septum in our center, performed with different nitinol wire mesh occluders with and without a protective layer. ${ }^{5}$ In this study, we included 74 subsequent adult patients with a history of percutaneous
ASD closure performed between 2015 and 2017. The study population was divided into 2 groups: 37 patients below 50 years of age (median [range] age, 34 [18-45] years) were included in group 1 and 37 patients above 50 years of age (median [range] age, 58 [50-72] years), in group 2. We collected data on selected clinical characteristics, heart catheterization, and echocardiography results (TABLE 1). The balloon-stretched diameter of ASD was measured in 24 patients from group 1 and 24 from group 2 . Applying a technique which was described elsewhere, ${ }^{6}$ the following atrial septal occluders were used: 40 Hyperion (Shanghai Shape Memory Alloy Co, Shanghai, China), 21 Heart R (Lifetech Scientific Corporation, Shenzen, China), 6 Cera (Lifetech Scientific Corporation, Shenzen, China), and 7 Cocoon (Vascular Innovations, Nonthaburi, Thailand) occluders. To minimize nickel release to serum, the following occluders have different protective layers: preoxidized nitinol in Hyperion, titanium nitride in Cera, and platinum in Cocoon. The Heart $\mathrm{R}$ occluder is produced without any protective layer on the nitinol wire. The application of the above mentioned devices depended on its availability (results of conducted tenders). Patients in whom the ASO (Abbott Medical, Plymouth, Minnesota, United States) and Figulla (Occlutech GmbH, Jena, Germany) occluders were used in this period were excluded from the study in order to ensure homogeneity of the sample. 
TABLE 1 Selected clinical characteristics, data on heart catheterization, and echocardiography (before closure) in adult patients in whom atrial septal defect was closed percutaneously at the age below 50 years (group 1) or above (group 2) using nitinol wire mesh occluders with and without a protective layer

\begin{tabular}{|c|c|c|c|c|}
\hline Parameter & All patients & Group $1(n=37)$ & Group $2(n=37)$ & $P$ value \\
\hline Age, y, median (min-max) & $47.5(18.9-72)$ & $34(18.9-45)$ & $58(50-72)$ & $<0.001$ \\
\hline Female sex, n (\%) & $57(77)$ & $28(75.7)$ & $29(78.4)$ & 0.78 \\
\hline ASD diametera, mm, mean (SD) & $17.6(5.6)$ & $17.42(5.8)$ & $17.67(5.5)$ & 0.86 \\
\hline $\begin{array}{l}\text { Diameter after stretching, mm, } \\
\text { mean (SD) }\end{array}$ & $21.4(5.49)$ & $21.6(5.7)$ & $21.17(5.36)$ & 0.79 \\
\hline Device diameter, mm, mean (SD) & $22.3(6.3)$ & $22.5(7)$ & $22.16(5.73)$ & 0.83 \\
\hline $\begin{array}{l}\text { Fluoroscopy time, min, median } \\
\text { (min-max) }\end{array}$ & $3.9(1.8-12)$ & $4(1.8-12)$ & $3.8(2-11)$ & 0.7 \\
\hline mPAP, mm Hg, mean (SD) & $18.1(5.56)$ & $16.32(4.83)$ & $20.28(5.72)$ & 0.007 \\
\hline $\mathrm{RVD}, \mathrm{cm}$, mean (SD) & $3.6(0.8)$ & $3.4(0.72)$ & $3.75(0.82)$ & 0.08 \\
\hline LVEDD, cm, mean (SD) & $4.4(0.55)$ & $4.32(0.55)$ & $4.47(0.54)$ & 0.25 \\
\hline RVD / LVEDD ratio, mean (SD) & $0.83(0.22)$ & $0.80(0.2)$ & $0.85(0.23)$ & 0.43 \\
\hline LVEF, \%, median (min-max) & $68(35-87)$ & $71(38-82)$ & $65(35-87)$ & 0.18 \\
\hline $\begin{array}{l}\text { Occluders without protection } \\
\mathrm{n}(\%)\end{array}$ & $21(28.4)$ & $10(27)$ & $11(29.7)$ & 0.8 \\
\hline Occluders with protectionc, n (\%) & $53(71.6)$ & $27(73)$ & $26(70.3)$ & 0.8 \\
\hline Periprocedural complications, $\mathrm{n}(\%)$ & $3(4.1)$ & $1(1.4)^{\mathrm{d}}$ & $2(2.7)^{\mathrm{e}}$ & 0.56 \\
\hline
\end{tabular}

a Measured on transesophageal echocardiography

b Occluders without a protective nitinol layer (Heart R)

c Occluders with a protective nitinol layer (Hyperion, Cera, Cocoon)

d A patient with an arteriovenous fistula

e One patient with an extensive hematoma at the puncture site and 1 patient with pericardial tamponade treated with pericardial drainage

Abbreviations: ASD, atrial septal defect; LVEDD, left ventricular end-diastolic diameter; LVEF, left ventricular ejection fraction; mPAP, mean pulmonary artery pressure; RVD, right ventricular diameter

Patients were scheduled for a routine check-up in the outpatient clinic 1, 6, and 12 months after ASD closure and yearly thereafter; 9 patients were lost to follow-up. The median (range) follow-up was 27 (4-39) months. The researchers contacted patients by phone to assess their physical condition using the New York Heart Association (NYHA) class. Patients' subjective view on that aspect was also elicited by asking, among others, the question "How do you evaluate your physical well-being after ASD closure?" with 5 possible answers: much better, better, the same, worse, much worse. The study protocol was approved by the institutional review board.

Statistical analysis Statistical analysis was performed with the use of the Statistica 12 software (TIBCO, Palo Alto, California, United States). $P$ values less than 0.05 were considered significant. Distribution of all variables was assessed with the Shapiro-Wilk test. Based on its results, the $t$ test or Mann-Whitney test was used to compare numerical variables. Categorical variables were compared using the $\mathrm{X}^{2}$ test.
Results and discussion Transcatheter ASD closure was successfully performed in 73 out of the 74 patients (98.6\%). In 1 patient from group 1 with relatively large ASD (treated with the 30-mm Hyperion occluder), the procedure was discontinued due to unfavorable morphology of the defect. The device was safely removed and the patient was referred for elective surgical closure of the defect.

While ASD (measured on transesophageal echocardiography) and applied devices' diameters were similar in both groups, mean pulmonary arterial pressure (measured directly in the catheterization laboratory) was higher in older patients (group 2). Left ventricular ejection fraction was lower in group 2 (the difference was not significant) as shown in TABLE 1.

All nitinol wire mesh occluders (with and without a protective layer) were similarly efficient with no difference in complication rate (no thrombosis, erosion, rupture, early or late embolization was observed). The fact that the delivery systems of Amplatzer-like occluders are 
generally 2-3 F larger than those of the ASO is less important in adults than in preschool children. ${ }^{1,5}$ The rates of periprocedural complications and procedural difficulties (reflected in the fluoroscopic time) were similar in both groups (TABLE1). In group 2, there were 3 patients with mild residual shunt diagnosed after the procedure by transthoracic echocardiography, which disappeared at 1-month follow-up. We observed substantial cardiac remodeling that involved a decrease in the right ventricular diameter (RVD) and right to left ventricular end-diastolic diameter ratio.

The mean (SD) decrease of the RVD was from $3.43(0.72)$ to $2.8(0.6) \mathrm{cm}$ in group 1 ( $t$ test, $P<0.05)$ and from $3.75(0.82)$ to $3.12(0.7) \mathrm{cm}$ in group 2 ( $t$ test, $P<0.05$ ). The right ventricular diameter (RVD) and right to left ventricular end-diastolic diameter ratio decreased from 0.8 (0.2) to $0.62(0.1)$ in group 1 ( $t$ test, $P<0.05$ ) and from $0.85(0.2)$ to 0.69 (0.21) in group 2 ( $t$ test, $P<0.05$ ).

After the procedure, the percentage of patients with NYHA class I raised from $28.6 \%$ to $82.1 \%$ in group 1 and from $9.5 \%$ to $66.7 \%$ in group 2.

Older patients (group 2) seemed to have a better subjective opinion about the change in their physical function than younger adults; $60.7 \%$ of the patients in group 1 felt "better" or "much better" after ASD closure, whereas the same answers were given by $76.2 \%$ of the patients in group 2 (difference not significant).

Although safety and effectiveness of ASD closure in the elderly has already been documented, ${ }^{6,7}$ all previous studies were based on the ASO devices. Moreover, our paper presents an undervalued and rarely described ${ }^{7}$ aspect of physical function, cardiac remodeling, and quality of life after ASD closure in this population. To our best knowledge, there is no report that compares the safety and efficacy of ASD closure in younger and older adults performed with so many different devices.

Nickel allergy is estimated to be near $10 \%$ in the general population. ${ }^{3}$ Cases requiring explantation of ASO were reported in the literature. ${ }^{4}$ We have not observed any symptoms of nickel allergy in our cohort, although we used occluders with bare nitinol wires (Heart R) as well as with a protective layer (Hyperion, Cera, Cocoon). Skin allergy to nickel does not equate adverse effects after device implantation and the mechanism of intracardiac hypersensitivity to nickel differs from skin allergy. ${ }^{8}$ However, based on reports on acute reactions requiring explantation of the device, ${ }^{4}$ we believe that testing a skin reaction to nickel (in form of a nitinol occluder worn as a necklace) in patients with known allergy is helpful in clinical practice. It may exclude patients with severe allergy who might develop an acute reaction to the occluder. We did not report a case requiring the explantation of a nitinol occluder due to nickel allergy; however, patients with severe allergy might benefit from the implantation of different Amplatzer-like occluders with protective layers.

Limitations The retrospective nature and the small number of patients are the main limitations of our study.

Conclusions Transcatheter closure of ASD in adult patients below and above the age of 50 years is a safe and effective procedure. The application of different Chinese and Thai nitinol wire occluders (with or without a protective layer) has similar effectiveness in this population.

\section{ARTICLE INFORMATION}

ACKNOWLEDGMENTS We would like to thank Weronika Duk and Katarzyna Potyka from the Student Research Group for their help in collecting the follow-up data.

CONFLICT OF INTEREST None declared.

OPEN ACCESS This is an Open Access article distributed under the terms of the Creative Commons Attribution-NonCommercial-NoDerivatives 4.0 International License (CC BY-NC-ND 4.0), allowing third parties to download articles and share them with others, provided the original work is properly cited, not changed in any way, distributed under the same license, and used for noncommercial purposes only. For commercial use, please contact the journal office at kardiologiapolska@ptkardio.pl.

HOW TO CITE Tyc F, Suchodolski A, Knop M, et al. Transcatheter closure of atrial septal defect with Chinese and Thai nitinol wire mesh occluders in adult patients. Kardiol Pol. 2020; 78: 68-70. doi:10.33963/KP.15121

\section{REFERENCES}

1 Knop MT, Białkowski J, Szkutnik M, et al. Transcatheter closure of atrial septal defects type 2 in children under three years of age. Kardiol Pol. 2018; 76: 1257-1262.

2 Lipiec $P$, Miśkowiec D, Peruga JZ, et al. Conscious sedation for transcatheter implantation of atrial septal occluders with two- and three-dimensional transoesophageal echocardiography guidance - a feasibility and safety study. Kardiol Pol. 2018; 76: 406-412.

3 Ries MW, Kampmann C, Rupprecht HJ, et al. Nickel release after implantation of the Amplatzer occluder. Am Heart J. 2003; 145: 737-741.

4 Spina R, Muller DWM, Jansz $P$, Gunalingam B. Nickel hypersensitivity reaction following Amplatzer atrial septal defect occluder device deployment successfully treated by explantation of the device. Int J Cardiol. 2016; 223: 242-243.

5 Białkowski J. Percutaneous closure of atrial septal defect type II - a few remarks on the basis of my own experience. Kardiol Pol. 2017; 26: 385-388.

6 Majunke N, Bialkowski J, Wilson N, et al. Closure of atrial septal defect with the Amplatzer septal occluder in adults. Am J Cardiol. 2009; 103: 550-554.

7 Komar M, Przewlocki T, Olszowska M, et al. The benefit of atrial septal defect closure in elderly patients. Clin Interv Aging. 2014; 9: 1101-1107.

$8 \mathrm{Kim} \mathrm{HJ}$, Shin JU, Lee J, et al. Positive reactions to nickel on a patch test do not predict clinical outcome of nickel alloy-based atrial septal defect occluder implantation. Dermatology. 2015; 230: 184-188. 